\title{
Search for ultra high energy primary photons at the Pierre Auger Observatory
}

\author{
Roberta Colalillo $^{1,2, a}$ for the Pierre Auger Collaboration ${ }^{3, b}$ \\ ${ }^{1}$ Universitäă Federico II di Napoli, Italy \\ ${ }^{2}$ INFN, Sezione di Napoli, Italy \\ ${ }^{3}$ Observatorio Pierre Auger, Av. San Martín Norte 304, 5613 Malargüe, Argentina
}

\begin{abstract}
The Pierre Auger Observatory, located in Argentina, provides an unprecedented integrated aperture in the search for primary photons with energy above $10^{17} \mathrm{eV}$ over a large portion of the southern sky. Such photons can be detected in principle via the air showers they initiate at such energies, using the complement of Auger Observatory detectors. We discuss the results obtained in diffuse and directional searches for primary photons in the EeV energy range.
\end{abstract}

\section{Introduction}

Photons are possible primary cosmic messengers in the flux of particles with energy larger than $10^{18} \mathrm{eV}$. The Pierre Auger Observatory and other experiments have observed a suppression in the cosmic ray energy spectrum at $E_{G Z K} \sim 6 \times 10^{19} \mathrm{eV}$. This feature could be related to the GZK effect $[1,2]$, but also to the photo-disintegration of heavy nuclei or to the maximum energy reached by the accelerating sources. The GZK effect predicts that cosmic rays with energies above the threshold energy $E_{G Z K}$ interact with cosmic microwave background photons, $\gamma_{C M B}$, and produce pions via the $\Delta$ resonance: $\gamma_{C M B}+p \rightarrow \Delta^{+} \rightarrow p+\pi^{0} ; \gamma_{C M B}+p \rightarrow \Delta^{+} \rightarrow n+\pi^{+}$. GZK photons come from the $\pi^{0}$ decay, hence the observation of these Ultra High Energy (UHE) photons would be a proof of the GZK cut-off and a way to constrain source and propagation models. Moreover, photons are not deflected in magnetic fields, so the study of UHE photons is important in searching for astronomical sources, and the presence or lack of UHE photons could have an impact on the measurements of the energy spectrum, cross sections, mass composition of cosmic rays, and on fundamental physical processes.

The Pierre Auger Observatory, located near Malargüe, Argentina, consists of a surface detector array (SD) [3] of 1660 water Cherenkov stations spread over an area of $3000 \mathrm{~km}^{2}$ and overlooked by 27 air fluorescence telescopes (FD) [4]. It is optimized to study Ultra High Energy Cosmic Rays (UHECR). The longitudinal profile of the showers is observed by the FD, while the distribution of shower particles at the ground is evaluated with the SD. Showers induced by photons develop deeper in the atmosphere compared to hadron showers, so the depth of shower maximum, $X_{\max }$, is larger for photon-induced events. In addition, they are also characterized by a smaller number of secondary muons and their signal in the SD

\footnotetext{
ae-mail: colalillo@na.infn.it

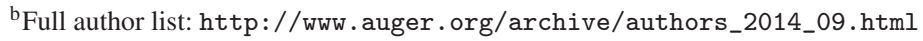


has peculiar characteristics. Using these properties of photon-induced showers, several upper limits on the photon fraction and flux were published by the Pierre Auger Collaboration.

\section{Upper limit on the cosmic ray photon flux using the surface detector}

Upper limits on the photon flux above 10, 20, and $40 \mathrm{EeV}$ were derived using data recorded by the surface detector [5]. The approach is based on observables sensitive to the longitudinal shower development and to the muon content, the signal risetime and the curvature of the shower front. The radius of curvature for photon-initiated showers is smaller than that for hadronic showers, but the risetime is larger for deeper showers. Data recorded from 2004 to 2006 were analyzed.

The criteria to select well-reconstructed events are: the station with the largest signal is surrounded by six active stations; $\geq 5$ stations are used in the fitting of the lateral distribution function out of which $\geq 4$ stations have a non-saturated signal of $\geq 10$ VEM (vertical equivalent muons); the reduced $\chi^{2}$ from the fit to estimate the radius of curvature is $<10$. The photon efficiency decreases to small values below $\sim 10 \mathrm{EeV}$. At higher energy, near-vertical photons can also fail the station multiplicity cut due to their deep development. Therefore, the analysis is restricted to primary energies $\geq 10 \mathrm{EeV}$ and primary zenith angles of $30-60^{\circ}$.

The first step in the search for photon candidates is the construction of the deviation $\Delta_{x}$ of the observable $x$ ( $x=t_{1 / 2}$ or $R$ referring to risetime or radius of curvature, respectively) from the mean value $\bar{x}_{\gamma}$ predicted for photons from simulated photon-initiated showers in units of the spread $\sigma_{x, \gamma}$ of the observable $x$ :

$$
\Delta_{x}=\frac{x-\bar{x}_{\gamma}(S(1000), \theta)}{\sigma_{x, \gamma}(S(1000), \theta)},
$$

where $S(1000)$ is the time-integrated energy deposit at $1000 \mathrm{~m}$ from the shower core and is used as an energy estimator.

Then $\Delta_{t_{1 / 2}}$ and $\Delta_{R}$ are combined to perform a principal component analysis [6]. A 5\% fraction of the real events, as well as the results from photon simulations are used to derive the principal component, actually the axis with the largest variance. The remaining $95 \%$ of the data are then projected onto the principal axis together with the data from simulated photons as shown in Fig. 1(a). The simulations have been performed with the AIRES package using the QGSJET hadronic interaction model, and a power law spectrum of index -2.0 has been assumed. In the projected distribution, a cut has to be established to select photon candidate events. The mean of the distribution for photons is chosen as the cut, so the efficiency of this cut is $f=0.5$ by construction. Any real event falling above this cut is considered a photon candidate.

Finally, an upper limit on the number of photons $N_{\gamma}^{C L}$ at the $95 \%$ confidence level $(C L)$ is calculated from the number of photon candidate events $N_{\gamma}$ above a minimum energy, $E_{\min }$. The upper limits on the flux and fraction of photons are based on $N_{\gamma}^{C L}$. The flux limit is given by:

$$
\Phi_{C L}\left(E>E_{\text {min }}\right)=\frac{N_{\gamma}^{C L}\left(E_{\gamma}>E_{\text {min }}\right) \times \frac{1}{f} \times \frac{1}{\epsilon}}{0.95 A},
$$

where $f=0.5$ is the photon selection cut efficiency, $\epsilon$ is the integrated efficiency of accepting photons and $A$ is the exposure of the detector. The factor 0.95 originates from the fact that only $95 \%$ of the data are used to determine the number of photon candidate events. The upper 


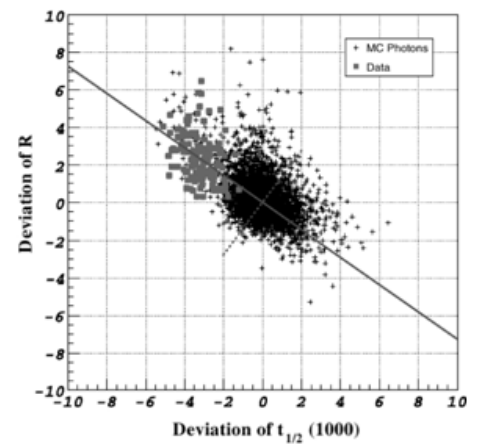

(a)

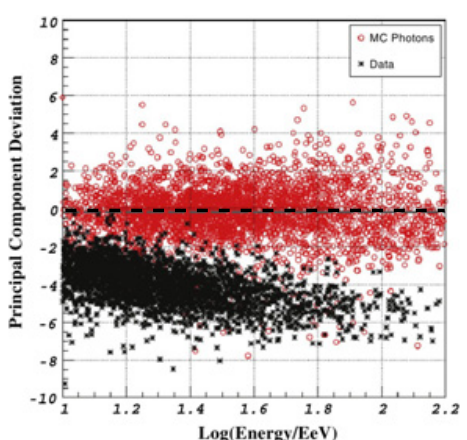

(b)

Figure 1. a) Deviation from a photon prediction for $5 \%$ of the data (closed squares) and for simulated photon showers (crosses). The solid grey line is the principal component while the dashed line is the axis perpendicular to the principal component. The minimum energy is $10 \mathrm{EeV}$. b) Deviation of data (black crosses) and photons (open red circles) from the principal component as a function of the primary energy (photon energy scale). The dashed line indicates the mean of the distribution for photons, chosen as the selection criterion. Data lying above this line are photon candidates. No event meets this requirement.

limit on the photon fraction is given by:

$$
F_{C L}\left(E>E_{\text {min }}\right)=\frac{N_{\gamma}^{C L}\left(E_{\gamma}>E_{\text {min }}\right) \times \frac{1}{f} \times \frac{1}{\epsilon}}{N_{\gamma}\left(E_{\gamma}>E_{\text {min }}\right)+N_{\text {non }-\gamma}\left(E_{\text {non }-\gamma}>E_{\text {min }}\right)},
$$

where $N_{n o n-\gamma}$ represents the number of non-photon candidate events in the data set for the fraction limit.

No event passes the photon candidate cut, as shown in Fig. 1(b). The upper limits on the photon flux above 10,20 , and $40 \mathrm{EeV}$ are then, respectively, $3.8 \times 10^{-3}, 2.5 \times 10^{-3}$, and $2.2 \times 10^{-3} \mathrm{~km}^{-2} \mathrm{sr}^{-1} \mathrm{yr}^{-1}$ (at the $95 \% C L$ ). The limits on the photon fraction are $2.0 \%$, $5.1 \%$, and $31 \%$ (at the $95 \% C L$ ) above 10,20 , and $40 \mathrm{EeV}$, respectively. The new limits are compared to previous results and to theoretical predictions in Fig. 3(b) for the photon flux and in Fig. 2 for the photon fraction. A discovery of a substantial photon flux could be interpreted as a signature of "top-down" models, which predict that UHECRs come from the decay of exotic particles directly at the energies observed. But these experimental limits put strong constraints on such models.

\section{Search for ultra high energy photons using hybrid events}

Hybrid events refer to air showers observed simultaneously with the surface array and fluorescence telescopes of the Auger Observatory, on dark, clear nights. From the calorimetric observation of the longitudinal development of ultra high energy air showers with the Auger Observatory, upper limits of 3.8\%, 2.4\%, 3.5\%, and $11.7 \%$ (at the 95\% CL) are obtained on the fraction of cosmic-ray photons above $2,3,5$, and $10 \mathrm{EeV}$, respectively [7]. These are the first experimental limits on ultra high energy photons below $10 \mathrm{EeV}$, so they complete the results obtained with data recorded by the surface detector (Sect. 2). The depths of shower maxima, $X_{\max }$, observed by fluorescence telescopes in hybrid mode was used to derive these upper limits. To improve the discrimination between photon-induced showers and showers initiated by protons, that are the hadronic showers most similar to photon-induced showers, and so to also improve the upper limits, another analysis was performed combining the $X_{\max }$ with a variable derived from the signal in the stations of the surface detector [8]. This variable, 


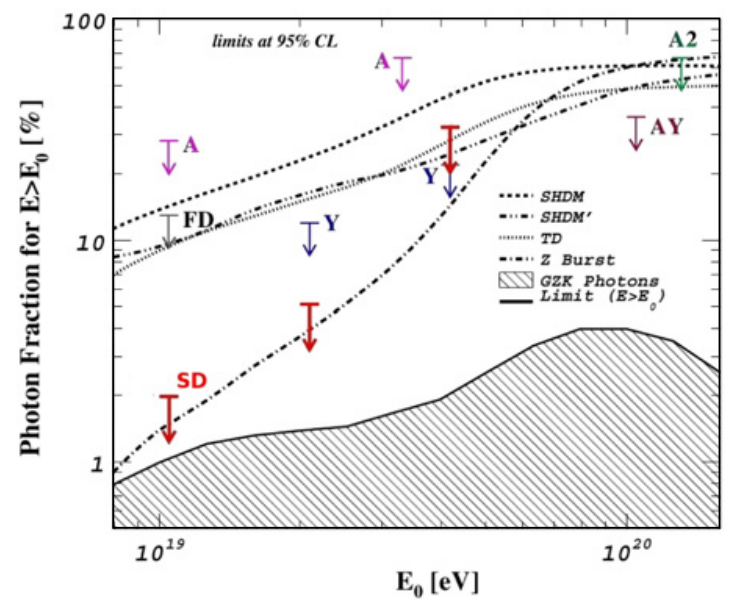

Figure 2. Upper limits on the fraction of photons derived in the work described in Sect. 2 (red arrows) along with previous experimental limits (A, A2: AGASA [11, 12], AY: AGASA-Yakutsk [13], Y: Yakutsk [14]; FD: Auger hybrid limit [16]). There is also the comparison with "top-down" models (TD, Z-Burst, SHDM from [17] and SHDM' from [18]).

called $S_{b}$, is defined as [9]:

$$
S_{b}=\sum_{i} S_{i}\left(\frac{R_{i}}{R_{\text {ref }}}\right)^{b},
$$

where the sum runs over the triggered stations, $S_{i}$ is the signal recorded by the $i$-th station placed at a distance $R_{i}$ from the hybrid shower core, and $R_{r e f}$ is a reference distance equal to $1000 \mathrm{~m}$ for this analysis. The exponent $b$ is chosen equal to 4 to maximize the separation power between photons and hadrons. $S_{b}$ is very sensitive to the difference in the muon component, and in fact combines the different amplitudes of the signals in the SD stations and the sharper distributions of the signal as a function of distance from the axis expected for photon-induced showers. In conclusion, these showers have a smaller $S_{b}$ than hadronic showers.

A Fisher analysis [10] is performed in bins of 0.5 in the logarithm of energy using photon and proton showers simulated with CORSIKA following a power law spectrum between $10^{17}$ and $10^{20} \mathrm{eV}$. QGSJET-II and FLUKA are used as hadronic interaction models. Events with zenith angle smaller than $60^{\circ}$ and with a good geometry reconstruction are selected for the analysis. The conditions to ensure a reliable profile reconstruction are: a reduced $\chi^{2}$ of the Gaisser-Hillas fit to the longitudinal profile smaller than 2.5 , the $\chi^{2}$ value obtained from a linear fit to the longitudinal profile exceeding the $\chi^{2}$ value from the Gaisser-Hillas fit by at least a factor of 1.1, the $X_{\max }$ observed within the field of view of the telescopes, any Cherenkov light contamination smaller than $50 \%$ and the uncertainty of the reconstructed energy less than $20 \%$. Moreover, only time periods with the sky not obscured by clouds and with a reliable measurement of the vertical optical depth of aerosols are used. To avoid an underestimation of $S_{b}$, that would mimic the behavior of a photon event, at least 4 active stations within $2 \mathrm{~km}$ from the hybrid reconstructed axis are required. The Fisher response for simulations with energy between $10^{18}$ and $10^{18.5} \mathrm{eV}$ (the first energy bin, the analysis is performed in three energy bins until $10^{19.5} \mathrm{eV}$ ) is shown in Fig. 3(a). The cut was chosen at the mean of the Fisher distribution for photons so the photon detection efficiency is $50 \%$ by 


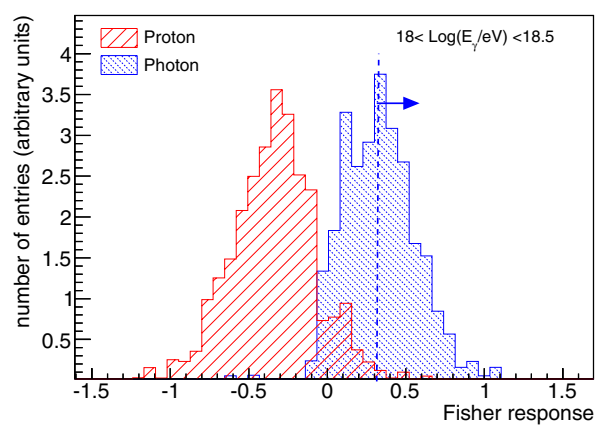

(a)

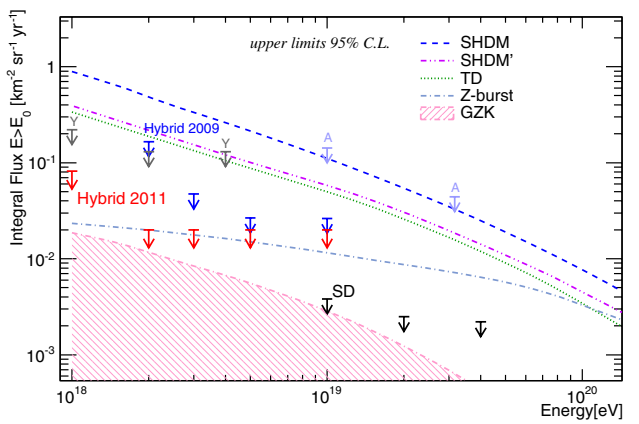

(b)

Figure 3. a) Fisher distribution for simulated protons (red) and photons (blue) with energy between $10^{18}$ and $10^{18.5} \mathrm{eV}$. Photon-candidate events have a Fisher value larger than $X_{\text {cut }}$ (dashed line, median of photon Fisher distribution). b) Upper limits on the photon flux above 1, 2, 3, 5, and $10 \mathrm{EeV}$ derived in the work described in Sect. 3 (red arrows) compared to previous limits from Auger (SD (work described in Sect. 2) and Hybrid 2009 [7]), from AGASA (A) [11] and Yakutsk (Y) [15]. The shaded region and the lines give predictions for the GZK photon flux [17] and for "top-down" models (TD, Z-Burst, SHDM from [17] and SHDM' from [18]).

construction. In the lowest energy interval, the expected hadron contamination is about $1 \%$ and it becomes smaller for increasing energies.

The data used for this analysis are hybrid showers collected between January 2005 and September 2010. Applying the cuts described above to data, 6, 0, 0, 0, and 0 photon candidates are found for energies above $1,2,3,5$, and $10 \mathrm{EeV}$, respectively, but the observed number of photon candidates is consistent with the expectation for nuclear primaries under the assumption of a mixed composition.

The $95 \% C L$ upper limits on the photon flux $\Phi_{\gamma}^{0.95}$ integrated above an energy threshold $E_{0}$ is given by:

$$
\Phi_{\gamma}^{0.95}=\frac{N_{\gamma}^{0.95}\left(E_{\gamma}>E_{0}\right)}{\epsilon_{\gamma, \min }},
$$

where $E_{\gamma}$ is the reconstructed energy using the photon energy scale, $N_{\gamma}^{0.95}$ is the number of photon candidates above $E_{0}$ at the $95 \% C L$, and $\epsilon_{\gamma, \min }$ is the minimum value (to be conservative) of the exposure of the hybrid detector. Upper limits on the integral photon flux of $8.2 \times 10^{-2} \mathrm{~km}^{-2} \mathrm{sr}^{-1} \mathrm{yr}^{-1}$ above $1 \mathrm{EeV}$ and $2.0 \times 10^{-2} \mathrm{~km}^{-2} \mathrm{sr}^{-1} \mathrm{yr}^{-1}$ above 2, 3, 5, and $10 \mathrm{EeV}$ are derived and are shown in Fig. 3(b). The results disfavor "top-down" models also in the lowest energy region. Using the Auger spectrum, upper limits on the fraction of photons of about $0.4 \%, 0.5 \%, 1.0 \%, 2.6 \%$, and $8.9 \%$ are obtained for energies above $1,2,3$, 5, and $10 \mathrm{EeV}$, respectively.

\section{Search for point sources of EeV photons}

The identification of photons coming from discrete sources is a direct way to identify the origins of cosmic rays; so an analysis was performed to search for point sources of EeV photons in the exposed sky using the hybrid events collected between January 2005 and September 2011 at the Pierre Auger Observatory [19]. The considered declination band goes from $-85^{\circ}$ to $+20^{\circ}$ and the energy range from $10^{17.3} \mathrm{eV}$ to $10^{18.5} \mathrm{eV}$. With this last choice, high event statistics is ensured and shower development processes that may introduce a bias 


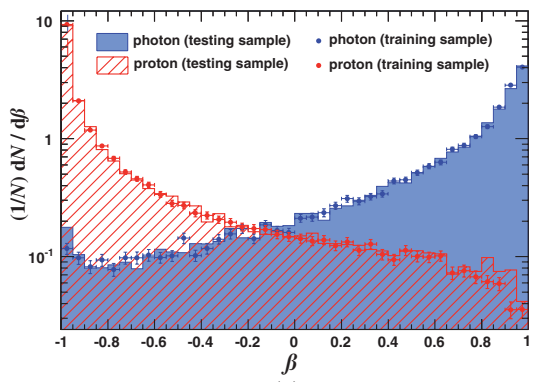

(a)

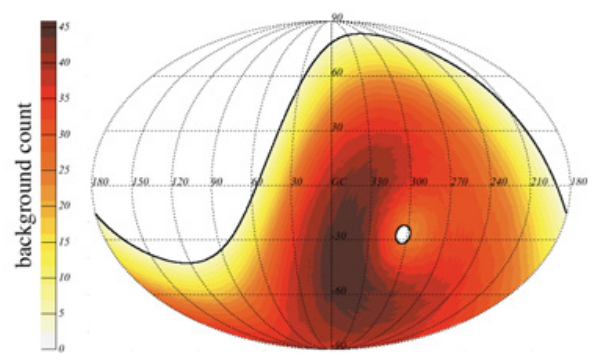

(b)

Figure 4. a) MVA output value, $\beta$, for photon and proton primaries using BDTs. The first two steps of this technique are training and testing, so the MC sample is split half into a training (filled circles) and half into a testing sample (solid line). b) Celestial map in Galactic coordinates of the expected background contribution. The solid black lines indicate the declination range covered in this analysis.

at highest energies (pre-shower and LPM [20]) are avoided. The goal of this analysis is to derive directional upper limits on the photon flux over all exposed celestial directions using a multivariate analysis, a Boosted Decision Tree (BDT) analysis [21, 22], in which FD and SD variables are combined to select photon candidate events.

The three selected FD variables are: the depth of shower maximum $X_{\max }$; the reduced $\chi^{2}$ from the fit to the longitudinal profile with a Greisen function (optimized to describe the longitudinal profile of pure elecromagnetic showers); and the ratio $E_{G r} / E_{G H}$, where $E_{G r}$ is the energy obtained with the Greisen fit and $E_{G H}$ is that obtained by the fit with the GaisserHillas function, the typical function used to describe the shower longitudinal profile.

The two SD observables used in the analysis are: $S_{b}$, already described in Sect. 3, but in the energy region of interest for this analysis, the exponent that maximizes the photon-hadron discrimination being $b=3$; and the shape parameter, that is the ratio of the early-arriving to the late-arriving integrated signal as a function of time measured in the SD stations with the strongest signal:

$$
\operatorname{ShapeP}(r, \theta)=\frac{S_{\text {early }}(r, \theta)}{S_{\text {late }}(r, \theta)} .
$$

The early and late signals are separated by a scaled time defined as:

$$
t_{i}^{\text {scaled }}(r, \theta)=t_{i} \cdot \frac{r_{0}}{r} \cdot \frac{1}{c_{1}+c_{2} \cdot \cos (\theta)},
$$

where $t_{i}$ is the real time of bin $i$ and $r_{0}$ is a reference distance equal to $1000 \mathrm{~m}$. Here $c_{1}=-0.6$ and $c_{2}=1.9$ are scaling parameters to average traces over different inclination angles.

The separation is the quantity that evaluates the performance of BDTs. It is zero when signal and background have identical shapes and it is one when there is no overlap between the two shapes. The training and testing steps of BDTs are performed with photon and proton showers simulated using CORSIKA version 6.900. They are generated according to a powerlaw spectrum of index -2.7 , using QGSJET01 as the high-energy and GHEISHA as the low-energy interaction models. The MVA output variable, $\beta$, is shown in Fig. 4(a). In this analysis, events with zenith angle smaller than $60^{\circ}$ are used. The necessary cuts to have a reliable profile reconstruction and a good reconstruction of $S_{b}$ are the same as the ones described in Sect. 3. The only difference is that it is not required that $X_{\max }$ have been observed within the field of view because the aim is to maximize the acceptance for photon showers. Measured data are used to estimate the expected isotropic background using the scrambling 

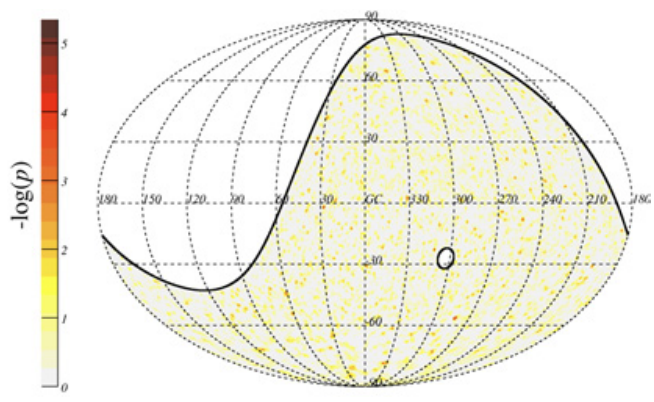

(a)

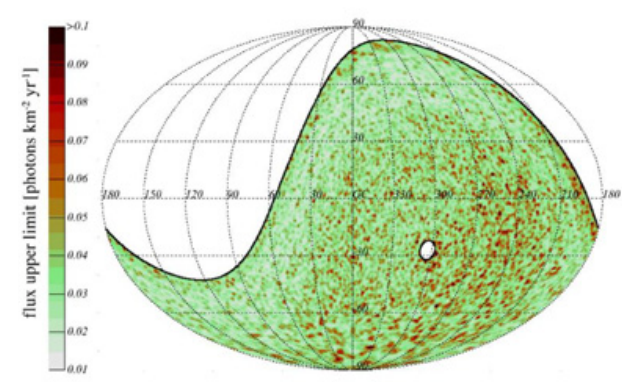

(b)

Figure 5. a) Sky map of $-\log (p)$ values in Galactic coordinates. b) Sky map of the photon flux in Galactic coordinates.

technique [23]. The sky map of directional background contribution for the covered search period is shown in Fig. 4(b).

To select photon candidate events, it is necessary to establish a cut on the $\beta$ variable. It is optimized by taking into account the expected number of background events $n_{b}(\alpha, \delta)$, which is a function of the celestial coordinates $\alpha$ (right ascension) and $\delta$ (declination). The upper limit of photons, $n_{s}$, from a point source in a given direction is calculated under the assumption that $n_{\text {data }}$, the observed number of events, is equal to the expected number of events that pass the $\beta$ cut, $n_{b}^{\beta}(\alpha, \delta)=n_{b}(\alpha, \delta) \cdot \epsilon_{\text {data }}^{\beta}(\delta)$ where $\epsilon_{\text {data }}^{\beta}$ is the data efficiency. This number is typically less than 4 events for the $\beta_{\text {cut }}$ values finally chosen. The procedure of Zech [24] is used to define the upper limit on the photon number at a given confidence level in the presence of a Poisson-distributed background:

$$
P\left(\leq n_{\text {data }}^{\beta} \mid n_{b}^{\beta}+n_{s}^{Z e c h}\right)=\alpha_{C L} \cdot P\left(\leq n_{\text {data }}^{\beta} \mid n_{b}^{\beta}\right)
$$

where $\alpha_{C L}=1-C L=0.05$ and $n_{b}^{\beta}$ is the expected background contribution. At this point, the probability $p$ of obtaining a test statistic has to be calculated: $p=\operatorname{Poiss}\left(\geq n_{\text {data }}^{\beta} \mid n_{b}^{\beta}\right)$ is the Poisson probability to observe $n_{\text {data }}^{\beta}$ or more events given a background expectation after $\beta_{\text {cut }}$ of $n_{b}^{\beta}$. The celestial map distribution of the $p$-value is shown in Fig. 5(a). The directional upper limit on the photon flux from a point source is defined as:

$$
f^{U L}=\frac{n_{s}^{\text {Zech }}}{n_{\text {inc }} \cdot \epsilon_{\beta}}
$$

where $n_{s}^{Z e c h}$ is the limit on the number of photons from a given direction, $\epsilon_{\beta}$ the directional acceptance from the same target at the $95 \% C L$ and $n_{\text {inc }}$ is a correction term. Figure 5(b) shows the celestial map of upper limits on the photon flux. The $p$-values are referred to a local probability that the data is in agreement with a uniform distribution. The minimum $p$ value observed is $p_{\min }=4.5 \times 10^{-6}$. The probability to observe this value anywhere in the sky is $p_{\text {chance }}=36 \%$. The mean value of the photon flux is 0.035 photons $\mathrm{km}^{-2} \mathrm{yr}^{-1}$. This value is not a signature of a directional photon flux, in fact for the median exposure, a flux of 0.14 photons $\mathrm{km}^{-2} \mathrm{yr}^{-1}$ or greater would yield an excess of at least $5 \sigma$.

\section{Conclusions}

The analyses performed until now by the Pierre Auger Collaboration to observe ultra high energy photons have not found candidates, but imposed severe constraints on "top-down" 
models. The GZK photons could be observed in the near future; the idea is to enhance the sensitivity with new analyses, an upgraded detector and accumulating more statistics.

Also the "blind analysis", that is the analysis performed in all parts of the exposed sky, to search for a directional photon flux has not detected any photon point sources. If some real photon fluxes are responsible for some of the lower $p$-values seen, additional exposure should increase the significance of those excesses. Moreover, targeted searches, choosing one or more directions of significance, are ongoing.

\section{References}

[1] K. Greisen, Phys. Rev. Lett. 16, 748-750 (1966)

[2] G.T. Zatsepin and V.A. Kuz'min, Journal of Experimental and Theoretical Physics Lett. 4, 78-80 (1966)

[3] The Pierre Auger Collaboration, Nucl. Instr. Meth. Phys. Res. A 613, 29-39 (2010)

[4] The Pierre Auger Collaboration, Nucl. Instr. Meth. Phys. Res. A 620, 227-251 (2010)

[5] The Pierre Auger Collaboration, Astropart. Phys. 29, 243-256 (2008)

[6] E. Oja, Int. J. Neural Syst. 1, 61-68 (1989)

[7] The Pierre Auger Collaboration, Astropart. Phys. 31, 399-406 (2009)

[8] M. Settimo for The Pierre Auger Collaboration, 32nd ICRC, Beijing 2011, HE1.3, 0393

[9] G. Ros et al., Astopart. Phys. 35, 140-151 (2011)

[10] R. A. Fisher, Annals Eugenics 7, 179-188 (1936)

[11] K. Shinozaki et al., Astrophys. J. 571, L117-L120 (2002)

[12] M. Risse et al., Phys. Rev. Lett. 95, 171102:1-4 (2005)

[13] G.I. Rubtsov et al., Phys. Rev. D 73, 063009:1-8 (2006)

[14] A. Glushkov et al., Journal of Experimental and Theoretical Physics Lett. 85, 163-167 (2007)

[15] A. Glushkov et al., Phys. Rev. D 82, 041101:1-5 (2002)

[16] The Pierre Auger Collaboration, Astropart. Phys. 27, 155-168 (2007)

[17] G. Gelmini, O. Kalashev, D. Semikoz, J. Exp. Theor. Phys. 106, 1061-1082 (2008)

[18] J. Ellis et al., Phys. Rev. D 74: 115003:1-11 (2006)

[19] The Pierre Auger Collaboration, Astrophys. J. 789, 160-171 (2014)

[20] M. Risse, P. Homola, Mod. Phys. Lett. A 22, 749-766 (2007)

[21] L. Breiman, J. Friedman, R. A. Olshen, and C. J. Stone, Classification and Regression Trees (Wadsworth and Brooks, Monterey (CA), 1984)

[22] R. E. Schapire, Mach. Learn. 5, 197-226 (1990)

[23] G. L. Cassiday et al., Nucl. Phys. Proc. Suppl. 14A, 291-298 (1990)

[24] G. Zech, Nucl. Instrum. Meth. A 277, 608-610 (1989) 